\title{
Food quality triggers the reproductive mode in the cyclical parthenogen Daphnia (Cladocera)
}

\author{
Ulrike Koch · Eric von Elert · Dietmar Straile
}

\begin{abstract}
Cyclic parthenogenesis (heterogony) is a widespread reproductive mode found in diverse taxa such as digenean trematodes, gall wasps, gall midges, aphids, cladocerans and rotifers. It is of particular interest as it combines the advantages of asexual reproduction (rapid population growth) and sexual reproduction (recombination). Usually sexual reproduction is initiated when, or slightly before, environmental conditions deteriorate, and often results in the production of resting stages. The optimal timing of diapause induction must thus be under strong natural selection. Using the cladoceran Daphnia as a model system, we show here for the first time that the switch from parthenogenetic to sexual reproduction in a cyclical parthenogenetic organism can be influenced by the chemical composition of food. Under crowding conditions Daphnia reproduced parthenogenetically with subitaneous eggs when fed the algal species Cryptomonas sp., but started the production of resting eggs when fed with the green algal species Scenedesmus obliquus. Supplementation experiments with lipids and especially proteins showed that the induction of resting egg production in two clones of
\end{abstract}

Communicated by Dag Olav Hessen.

Electronic supplementary material The online version of this article (doi:10.1007/s00442 0081216 6) contains supplementary material, which is available to authorized users.

U. Koch $(\bowtie) \cdot$ E. von Elert · D. Straile

Limnological Institute, University of Konstanz,

Mainaustrasse 252, 78464 Constance, Germany

e mail: ully koch@gmx.de

Present Address:

E. von Elert

Zoological Institute, Workgroup Aquatic Chemical Ecology,

University of Köln, Weyertal 119, 50931 Cologne, Germany different Daphnia species was due to a dietary deficiency in the green alga. Hence, the low food quality induced a switch in the reproductive mode that may contribute to optimal timing of the sexual reproduction of Daphnia in nature. Furthermore, our results have two other major implications: first, they suggest that protein compounds should be added to the list of diet constituents potentially limiting or influencing Daphnia reproduction. Second, we show that the role of food quality goes far beyond the up to now documented effects of food quality on somatic growth and trophic transfer efficiency of herbivores: due to its effects on sexual reproduction and the production of resting eggs, food quality might influence genetic diversity and long-term persistence of Daphnia in lakes.

Keywords Cyclical parthenogenesis .

Resting egg production - Daphnia - Food quality ·

Sexual reproduction

\section{Introduction}

Within sexually reproducing species and populations the production of male gametes results in a factor of 2 reduction of fitness, as males themselves do not produce offspring, and energetic resources are required for their production (see Maynard Smith 1978). Cyclical parthenogenetic animals have evolved a reproductive mode that largely overcomes this cost while maintaining the benefits of sex, i.e. genetic reshuffling (Peck et al. 1999). In many cyclical parthenogenetic organisms, sexual production confers an additional benefit: the production of resting eggs. For example, sexually produced eggs of aphids are able to resist freezing (Simon et al. 2002) and hence are of key importance for the persistence of aphid populations in 
colder regions. In the key herbivore Daphnia, sexually produced resting eggs (ephippia) are resistant to freezing and desiccation (Schwartz and Hebert 1987). Additionally, ephippia can survive for several decades and hence are important for dispersal in space (Louette and De Meester 2005) and time (Hairston and Kearns 2002).

Like most other cyclical parthenogenetic animals, Daphnia often start to reproduce sexually when environmental conditions deteriorate (Stross and Hill 1965; Hobæk and Larsson 1990; Kleiven et al. 1992). During most of the season Daphnia reproduces parthenogenetically with subitaneously developing eggs, and sexual reproduction is restricted to specific times of the year, e.g. during short periods in early summer or autumn and winter (Cáceres 1998; Jankowski and Straile 2004). This indicates that the high costs associated with sexual reproduction and resting egg production have favoured the evolution of an inducible rather than a fixed switch from parthenogenetic to sexual reproduction. Accordingly, sexual reproduction in Daphnia has been shown to be inducible by a multitude of environmental factors such as population density, food concentration, photoperiod and fish kairomones (Stross and Hill 1965; Hobæk and Larsson 1990; Kleiven et al. 1992; Slusarczyk 1995), as well as by maternal environmental conditions (Alekseev and Lampert 2001; LaMontagne and McCauley 2001). This ensures that the onset of sexual reproduction is coupled to deteriorating abiotic and biotic environmental conditions for parthenogenetic reproduction.

The role of food quality in determining the reproductive mode in cyclical parthenogenetic organisms is not well understood. However, in the polymorph rotifer Asplanchna it has been shown that the presence of dietary $\alpha$-tocopherol (vitamin E) leads to the production of mictic female morphotypes (Gilbert and Thompson 1968; Gilbert 1981), which in turn produce resting eggs. Hence, in this system, the influence of food quality on the reproductive mode is indirect, as resting egg production depends on the female morphotype, the development of which is vitamin E dependent (Gilbert 2007). In aphids food quality has also been suggested to be important for the reproductive mode. However, up to now strong evidence for a role of food quality in producing resting eggs could not be found (Dixon and Kundu 1998; Dixon et al. 1993).

A major factor that determines growth and reproduction of Daphnia in nature is the composition of its food, which undergoes pronounced seasonal changes due to the succession of algal species and seasonally varying ratios of algal to detritus biomass (Sommer 1985). In early spring, lakes and ponds develop an algal maximum composed of small diatoms and fast-growing flagellates, i.e. Cryptomonas sp. that are of high food quality for Daphnia, while in summer other phytoplankton taxa, among them green algae, occur and lead to a lower food quality. During early summer, i.e. during the clear-water phase, food quality seems to be especially low (Wacker and Von Elert 2001) presumably due to a low contribution of algae to seston relative to detritus (D. Straile, unpublished data). Early summer is a preferred time for sexual reproduction in Daphnia (Jankowski and Straile 2004). This has been suggested to be due to high Daphnia densities (e.g. Stross and Hill 1965; Hobæk and Larsson 1990; Kleiven et al. 1992) and/or declining food availability (LaMontagne and McCauley 2001). Up to now, food quality has not been considered as a cue contributing to the decision of Daphnia to switch its reproductive mode in early summer. However, the strong change in food quality observed during this time period (Wacker and Von Elert 2001), suggests that a decrease in food quality may at least contribute to ensure the proper timing of the switch from parthenogenetic to sexual reproduction. To test the influence of the biochemical composition of food algae on Daphnia's reproductive mode, we fed two clones belonging to two different Daphnia species (Daphnia galeata and Daphnia pulex) in laboratory experiments with two algal species that are known to be of minor (Scenedesmus obliquus) and superior (Cryptomonas sp.) food quality for Daphnia (Ahlgren et al. 1990, Weers and Gulati 1997).

\section{Materials and methods}

\section{Experimental animals and set up}

The study was conducted with one clone of D. galeata and one clone of $D$. pulex, both with a high allocation to ephippia production. The D. galeata clone is a sexual clone established from an ephippium which was taken from a sediment core sampled in Lake Constance, a large and deep pre-alpine lake in central Europe. The obligate parthenogenetic clone of $D$. pulex was originally isolated from a pond in northern Germany. Both Daphnia clones were cultured for many generations under laboratory conditions. The selected clones are particularly suitable for experiments on induction of resting egg production as they produce high amounts of ephippia even under slight crowding conditions, making additional treatments to enhance the usually very low ephippia production unnecessary. Experiments were run under continuous dim light at $20^{\circ} \mathrm{C}$ and with non-limiting food concentrations $\left(2 \mathrm{mg} \mathrm{C}^{-1}\right)$ in jars with $200 \mathrm{ml}$ filtered $(0.45-\mu \mathrm{m}$ membrane cartridge; Sartorius) lake water, including ten animals each, to simulate moderate crowding conditions. Previous experiments have shown that this density results 
in ephippia production in both clones. Mothers of the experimental animals were cultured under non-limiting food conditions ( $S$. obliquus, $2 \mathrm{mg} \mathrm{C}^{-1}$ ) for at least two generations. Each treatment was run with four replicates. Experimental animals were collected within $12 \mathrm{~h}$ after birth. Only third-clutch individuals were used. Ephippia production experiments lasted from birth to 16 days of age. During this time period, the animals could produce three parthenogenetic/subitaneous or two ephippial broods. During the experiment, the animals were fed in different treatments with the following types of food: $S$. obliquus (2 $\left.\mathrm{mg} \mathrm{C}^{-1}\right)$; Cryptomonas sp. (2 $\left.\mathrm{mg} \mathrm{C}^{-1}\right)$; S. obliquus $\left(2 \mathrm{mg} \mathrm{C}^{-1}\right)+$ protein beads $\left(30 \mu \mathrm{l} 200 \mathrm{ml}^{-1}\right)$, S. obliquus $\left(2 \mathrm{mg} \mathrm{C}^{-1}\right)+$ protein beads $\left(30 \mu \mathrm{l} 200 \mathrm{ml}^{-1}\right)$ loaded with Cryptomonas sp. lipid. The animals were transferred daily into fresh medium (food suspension), whereby released ephippia and neonates were counted and separated from the experimental animals. This procedure excludes the possibility of ephippia fertilization by males, and resulted in empty ephippia for the sexual clone. Allocation to sexual reproduction/resting egg production was measured as number of released ephippia. Allocation to parthenogenesis/subitaneous eggs was measured as total number of released neonates. In the following we use "parthenogenesis" for subitaneous developing offspring numbers and "ephippia production" for resting egg numbers of both clones. Male production was not recorded, as it could only be expected in the D. galeata clone. Furthermore, the induction of males is reported to be presumably induced by levels or combinations of cues slightly different from those inducing ephippia production (Hobæk and Larsson 1990). Hence, the role of food quality for male production in Daphnia needs additional study.

\section{Algae cultures}

The green alga S. obliquus (SAG 276-3; Stammsammlung für Algen, Göttingen, Germany) was grown in batch culture in Cyano medium (Jüttner et al. 1983$)\left(20^{\circ} \mathrm{C}\right.$, illumination $146 \mu \mathrm{mol}$ quanta s $\mathrm{s}^{-1} \mathrm{~m}^{-2}$ ). The cryptophyte Cryptomonas sp. (SAG 28.80) was grown in semi-continuous culture (dilution 0.2 day $^{-1}$ ) in WC medium with vitamins (Guillard 1975). P supply for algae in both media was non-limiting, thus preventing the possibility of P-limitation of Daphnia.

Algae sedimentation, a potential means of food limitation in the jars after $24 \mathrm{~h}$, was analysed once in a test using a CASY particle counter. These measurements suggested that food limitation at the end of the $24 \mathrm{~h}$ period, if any, was only minor. In fact, only large $D$. pulex in combination with sedimentation were able to suppress food availability at the end of the experiment to below $0.5 \mu \mathrm{g} \mathrm{C} 1^{-1}$. However, D. pulex as a pond species is likely to feed also on sedimented algae. Hence, besides crowding, all other known stimuli for resting egg production were excluded, i.e. strong food limitation, fish kairomones or changes in photoperiod.

\section{Supplementation experiments}

To get more information about the chemical nature of the substance preventing the switch to ephippia production, we supplemented S. obliquus with extracted Cryptomonas sp. lipid attached to protein (bovine serum albumin; BSA) 6- $\mu \mathrm{m}$-diameter beads (Micromod, Rostock, Germany), according to Von Elert and Wolffrom (2001). For lipid extraction, $30 \mathrm{mg} \mathrm{C}$ of Cryptomonas sp. algae was filtered onto pre-combusted glass fibre filters (Whatman GF/F). Lipids were extracted with dichloromethane/methanol (2:1; $\mathrm{v}: \mathrm{v})$, dried and dissolved in $10 \mathrm{ml}$ absolute ethanol for storage. Protein beads were stored as stock solution in absolute ethanol $\left(20 \mathrm{mg} \mathrm{ml}^{-1}\right)$. For supplementation with pure protein beads, $30 \mu \mathrm{jar}^{-1}$ of the stock solution was diluted in $30 \mathrm{ml}$ absolute ethanol, dried and suspended in filtered lake water prior to the addition to algal food suspension. For protein beads loaded with Cryptomonas sp. lipid, $30 \mu \mathrm{jar}^{-1}$ of BSA beads was diluted in $30 \mathrm{ml}$ absolute ethanol, $400 \mu \mathrm{l}$ of the Cryptomonas sp. lipid extract (equal to the lipid amount of $1.2 \mathrm{mg} \mathrm{C}$ of Cryptomonas sp.) was added and both were dried and dissolved in filtered lake water.

\section{Estimation of Daphnia growth rates}

Daphnia performance on the different diets was further analysed by determining juvenile somatic growth rates $(g)$ and population growth rates $(r)$ calculated with Euler's equation. The latter provides a reasonable estimation of fitness for Daphnia (Stearns 1992) and is influenced directly by food quality and also indirectly due to switches in reproductive mode, i.e. from parthenogenetic to resting egg production. Juvenile $g$ is often used as a surrogate for $r$ (Lampert and Trubetskova 1996), but it will only be influenced directly by food quality as it is determined before reproductive decisions take place. The juvenile $g$ was calculated from standardized experiments (Wacker and Von Elert 2001) using the equation:

$g=\left[\ln \left(W_{5}\right)-\ln \left(W_{0}\right)\right] t^{1}$

where $W_{0}$ is the individual dry mass at the beginning of the experiment and $W_{5}$ is the individual dry mass after a growth period of 5 days. Dry masses were estimated as mean values of 1015 individuals weighed on an electronic microbalance (Mettler UMT 2) and recorded to the nearest $0.1 \mu \mathrm{g}$. 
$r$ was calculated using the Euler Lotka equation:

$1=\sum_{x=0}^{n} l_{x} m_{x} \mathrm{e}^{r x}$

with age-specific survival probabilities, $l_{x}$, and fecundities, $m_{x}$. Data were analysed using one-way ANOVA with post hoc comparisons (Tukey's HSD), and repeated measures (RM) ANOVA. Normality of data was checked using the Shapiro-Wilk's test and homogeneity of variances using Cochran and Bartlett's test. As numbers of ephippia produced per individual were not normally distributed we used ANOVA on ranks for this (e.g. Conover and Iman 1981).

Finally, we conducted a literature search using the Web of Science data base (Thomson Cooperation) to determine the food quality applied in earlier experimental work on Daphnia ephippia induction. For details see Electronic supplementary material Appendix 1.

\section{Results}

$r$ of Daphnia fed with the high-quality food Cryptomonas sp. were increased significantly for both Daphnia species compared to $S$. obliquus (Fig. 1a, c; Tukey's HSD $P<0.001$, following one-way ANOVA, $F_{3,11}=49.956$; $P<0.001$ for D. galeata; Tukey's HSD $P<0.001$, following one-way ANOVA, $F_{3,10}=88.771 ; P<0.001$ for D. pulex). This was, though to a smaller extent, also reflected in the juvenile $g$ (Fig. 1b, d; Tukey's HSD $P<0.05$, following one-way ANOVA, $F_{3,8}=47.044$; $P<0.001$, for D. galeata; Tukey's HSD $P<0.001$, following one-way ANOVA, $F_{3,12}=16.054 ; P<0.001$ for D. pulex).
However, the estimation of fitness according to Euler is based on parthenogenetic or subitaneously developing offspring and is hence sensitive to switches from parthenogenetic to sexual reproduction, i.e. resting egg production. Low population growth on $S$. obliquus coincided with substantially reduced numbers of parthenogenetic offspring for both Daphnia species when compared to animals fed Cryptomonas sp. (Fig. 2b, d; Tukey's HSD $P<0.001$, following one-way ANOVA, $F_{3,12}=37.484 ; P<0.001$ for $D$. galeata; Tukey's HSD $P<0.001$, following one-way ANOVA, $F_{3,11}=32.744$; $P<0.001$ for $D$. pulex). Overall offspring numbers were higher for $D$. pulex due to its larger size. The reduction of parthenogenetic offspring by the low-quality food $S$. obliquus was due to the onset of resting egg production: Daphnia fed with Cryptomonas sp. did not produce any ephippia, whereas ephippia production on S. obliquus was high for both Daphnia species (Fig. 2a, c; Tukey's HSD $P<0.001$, following one-way ANOVA, $F_{3,12}=28.941$; $P<0.001$ for D. galeata; Tukey's HSD $P<0.001$, following one-way ANOVA, $F_{3,10}=22.966 ; P<0.001$ for D. pulex). Hence, Daphnia's reproductive allocation depended strongly on algal species, i.e. food quality.

In order to confirm that the induction of resting egg production in Daphnia by $S$. obliquus was due to the absence of a compound that was a constituent of Cryptomonas sp. and that was responsible for the higher fitness of both Daphnia species on Cryptomonas sp. than on $S$. obliquus, we supplemented $S$. obliquus with protein (BSA) beads, and for additional lipid supplementation, with protein beads that had been loaded with Cryptomonas sp. lipids.

In both Daphnia species the supplementation of S. obliquus with protein lead to a substantial reduction in ephippia
Fig. 1 Population growth rate $(r)$ and somatic juvenile somatic growth rate $(g)$ for the two experimental clones belonging to Daphnia galeata and Daphnia pulex on four food treatments: Cryptomonas sp $(C)$, Scenedesmus obliquus $(S)$, S. obliquus + protein + Cryptomonas sp. lipid (SPL), S. obliquus + protein $(S P)$, means of four replicates $\pm \mathrm{SE}$. Average $r$ of D. galeata on S. obliquus was negative ( 0.2 , not shown). Bars labelled with different letters are significantly different based upon Tukey's HSD, at least $P<0.05$, following ANOVA
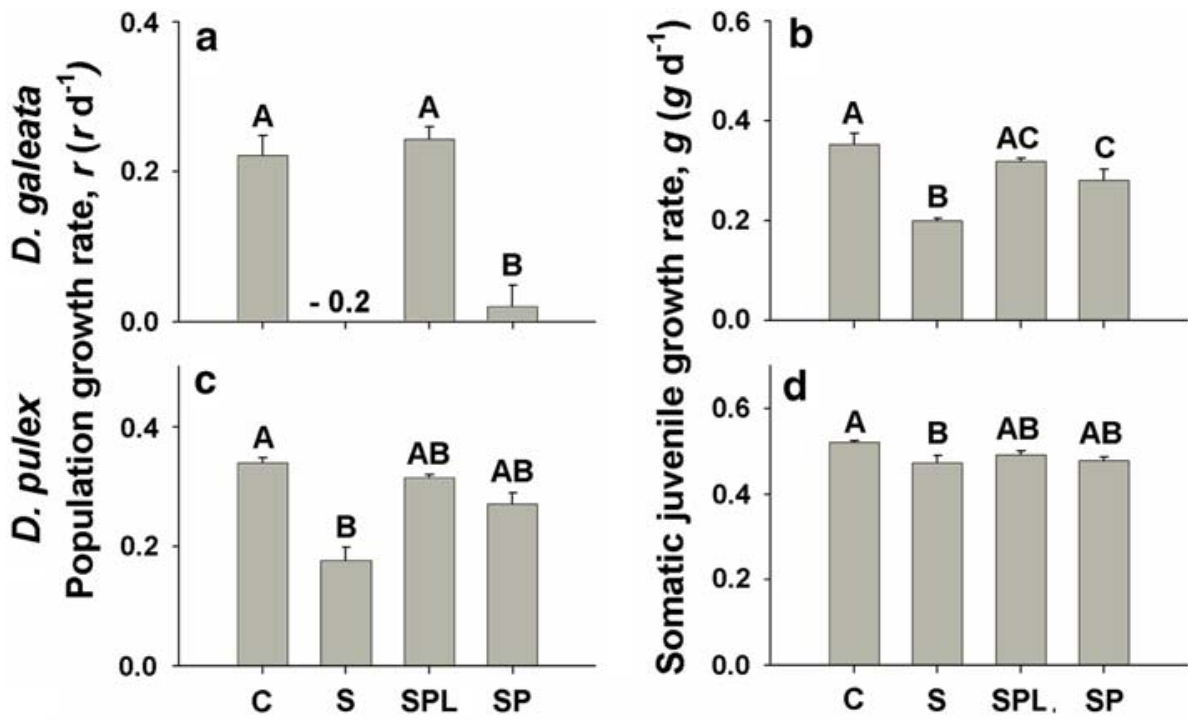

Food treatment 
Fig. 2 Parthenogenetic reproduction (expressed as neonate production) and ephippia production in the two experimental clones belonging to D. galeata and D. pulex on four food treatments: C, S, SPL, SP; means of four replicates \pm SE. Neonate and ephippia numbers are shown as sum per individual over 16 days after birth. First reproduction was on day 7 or 8 . Bars labelled with different letters are significantly different, based upon Tukey's HSD, at least $P<0.05$, following ANOVA. For abbreviations, see Fig. 1
Parthenogenetic reproduction
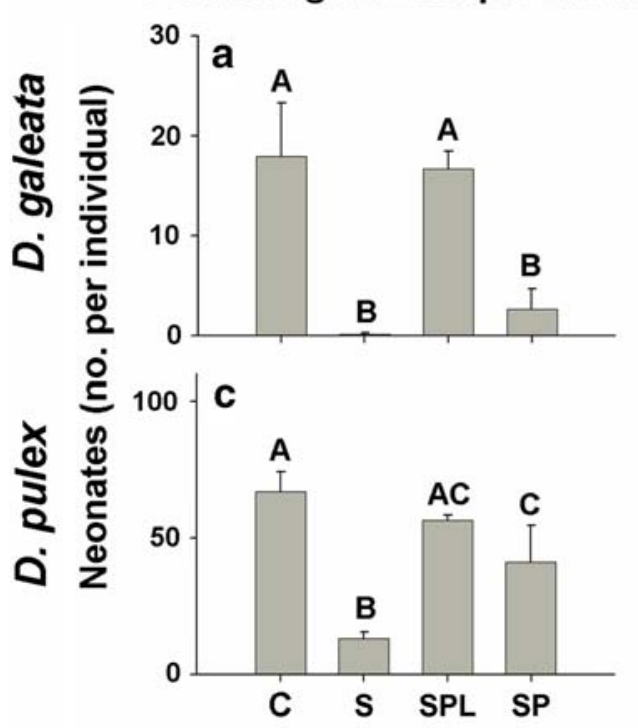

Ephippia production

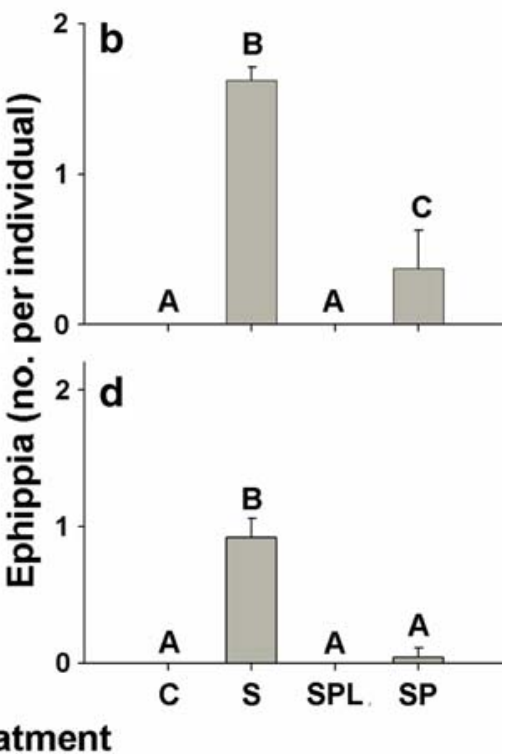

production (Fig. 2), demonstrating that a dietary deficiency and not the presence of toxins or inhibitors in S. obliquus induced ephippia production. However, only when the lowquality food, S. obliquus, was supplemented with both protein and Cryptomonas sp. lipids, was the same reproductive allocation as on pure Cryptomonas sp. observed, i.e. only parthenogenetic reproduction (Fig. 2; Tukey's HSD $P<0.001$, following one-way ANOVA, $F_{3,12}=$ 28.941; $P<0.001$ for $D$. galeata; Tukey's HSD $P<0.001$, following one-way ANOVA, $F_{3,10}=22.966$; $P<0.001$ for $D$. pulex, comparison to $S$. obliquus).

In addition, protein supplementation caused a significant increase in juvenile $g$ in $D$. galeata (Tukey's HSD $P<0.05$, following ANOVA, $F_{3,8}=47.044, P<0.001$ ), whereas this effect was less clear in $D$. pulex. A similar difference between the respective clones of each species, was observed with respect to $r$. Furthermore, the two species/clones differed also in the effect of lipid supplementation on $r$ : only lipid supplementation, not protein supplementation, significantly enhanced $r$ in D. galeata.

Figure 3 shows the time course of reproduction in both Daphnia species. On a Cryptomonas sp. diet both species produced three parthenogenetic clutches during the experiment. On $S$. obliquus the first clutch of both species consisted of an ephippium. While D. galeata continued ephippia production throughout the experiment, most D. pulex switched to parthenogenetic production after releasing one ephippium. Hence, the much stronger reduction in $r$ of D. galeata relative to $D$. pulex (Fig. 1) results from $D$. pulex's but not $D$. galeata's switch in reproductive mode with age (RM ANOVA: time $\times$ treatment $\times$ species interaction: $P<0.001)$. Supplementation with proteins caused a strong reduction in ephippia numbers, i.e. already in ephippial females for the first clutch in both Daphnia species. Some D. galeata still produced an ephippium with their second clutch. The numbers of parthenogenetically produced neonates remained, however, low in both clutches for $D$. galeata and $D$. pulex. Additional supplementation of lipids increased neonate numbers and resulted in a complete switch towards parthenogenetic reproduction in both Daphnia species. Thus the reproductive decision of Daphnia depended on a clonal-specific complex interplay between age and food quality.

\section{Discussion}

The role of food quality for Daphnia growth and consequently for the trophic coupling between phytoplankton and the key herbivore of many lake ecosystems is of considerable theoretical and applied interest (Müller-Navarra et al. 2000). Up to now, polyunsaturated fatty acids (Müller-Navarra et al. 2000; Von Elert 2002), sterols (Von Elert et al. 2003) and the mineral (P) content (Urabe et al. 1997) of algae have been considered as important facets of biochemical food quality for Daphnia. The algal species used in this study, Cryptomonas sp. and S. obliquus, are known to differ in their food quality for Daphnia. These food quality differences were thought to be particularly due to the fatty acid composition (Ahlgren et al. 1990), especially to the higher content of eicosapentaenoic acid (C20:5 $\omega 3$ ) in Cryptomonas sp. However, there is evidence that the cryptophyte Rhodomonas sp., a close relative of Cryptomonas, also shows a higher total content of amino acids compared to S. obliquus (Ahlgren et al. 1992). The importance of lipids for Daphnia is also evident in our 
Fig. 3 Parthenogenetic reproduction (expressed as neonate production) and ephippia production (number released per individual) in the two experimental clones belonging to $D$. galeata and D. pulex on four food treatments: a, b C; $\mathbf{c}, \mathbf{d ~ S} ; \mathbf{e}, \mathbf{f}$ SPL; $\mathbf{g}$, h SP; means of four replicates \pm SE. Left hand axes Neonates per individual, right hand axes ephippia per individual. For abbreviations, see Fig. 1
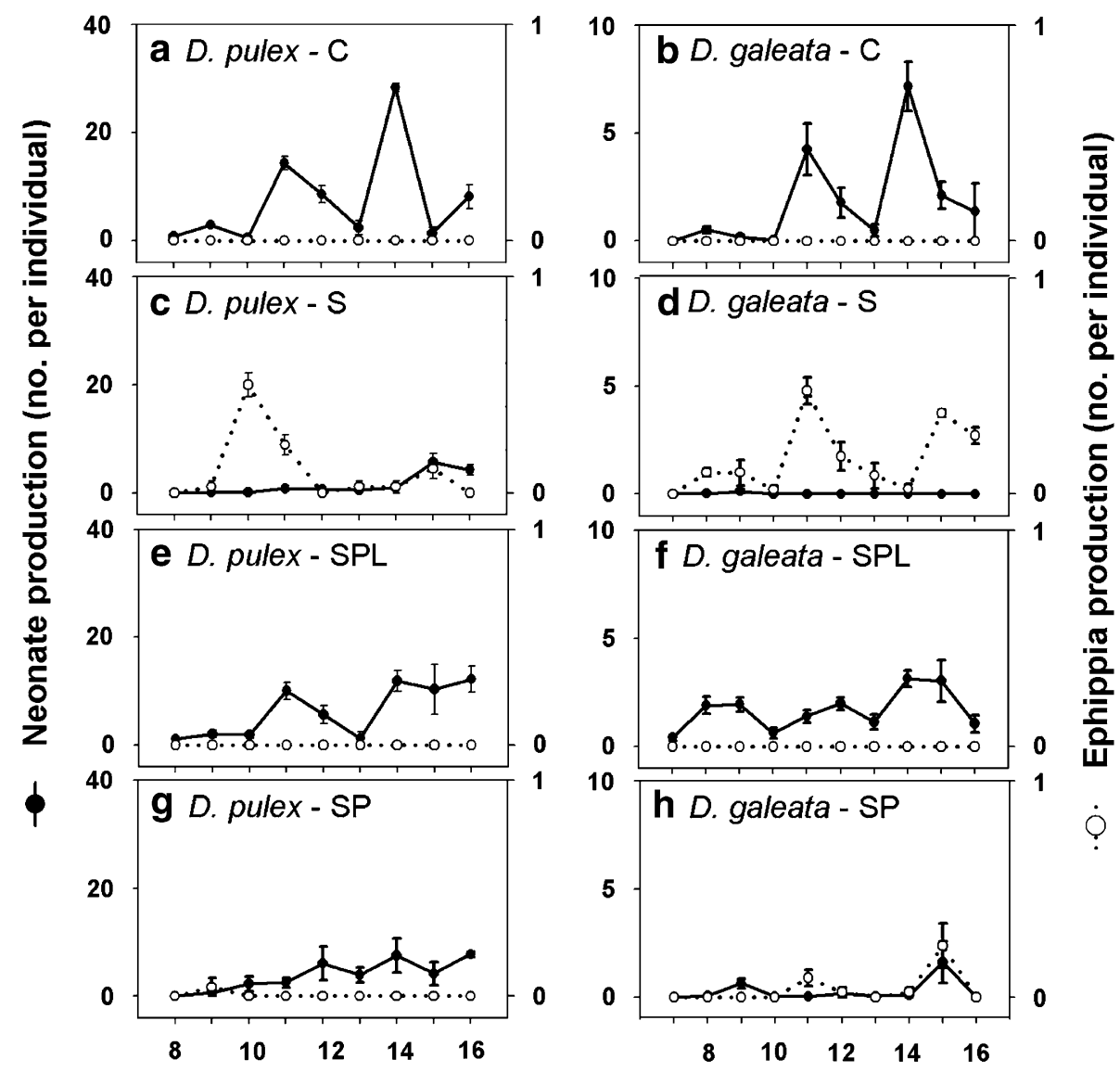

Time (d) experiments: especially in D. galeata, lipid supplementation strongly increased neonate production and accordingly $r$. However, our results suggest that additionally proteins can be important for $r$ and $g$ of Daphnia and its persistence in ecosystems. While previous studies did not report an effect of proteins (BSA) on Daphnia $g$ when supplemented with a cyanobacterial diet (e.g. Von Elert and Wolffrom 2001), we present evidence that proteins can increase $g$ at least when provided as a supplement to $S$. obliquus. The effect of BSA supplementation on $g$ was especially evident in D. galeata, while no clear effect could be demonstrated for $D$. pulex. However, $g$ of $D$. pulex on $S$. obliquus was only slightly below its $g$ on the high-quality food Cryptomonas sp., suggesting that $D$. pulex in contrast to D. galeata was hardly affected by the lower food quality of S. obliquus. Consequently, protein + lipid supplementation further increased the population increase in $D$. galeata but not in D. pulex. However, additional experiments with several D. galeata and D. pulex clones are needed to test if the differences observed are indeed differences at the species level or just clonal ones. Nevertheless, protein supplementation in both species/clones clearly resulted in a reduced ephippia and increased neonate production, i.e. in a switch between reproductive modes.
The food quality-mediated switch from subitaneous to resting eggs due to the protein content will clearly decrease Daphnia population growth and consequently also trophic transfer from algae towards higher trophic levels. On the other hand, this switch may increase Daphnia genetic diversity, secure Daphnia long-term survival within a lake or increase dispersal between lakes. All other things being equal, our results predict higher rates of Daphnia sexual reproduction in lakes and ponds with a low food quality. This would increase the chance for microevolutionary adaptation to low food quality, which has been suggested to have occurred in Lake Constance during eutrophication (Hairston et al. 1999). Likewise, increased ephippia production in lakes with low quality food would increase the possibility for dispersal into better food quality habitats. Altogether, this suggests that the role of proteins as a component of Daphnia food quality may be as important for lake functioning and Daphnia population dynamics as the food quality components considered up till now (see above). Clearly, there is an urgent need for more detailed studies on the specific components responsible for the protein effect in this study.

In nature only a few percent of female Daphnia carry ephippia even during times of maximal ephippia 
production. Hence, we chose crowding conditions and two Daphnia clones which invest strongly in ephippia production to enhance the incidence of ephippia in our experiments. Crowding conditions often prevail in situ, when Daphnia produce ephippia (Cáceres 1998; Jankowski and Straile 2004), and there is genetic variability in the propensity of Daphnia clones to invest in sexual reproduction (Deng 1996). Possibly, this variability arises at least partially from clonal variability in the sensitivity to food quality. One of our clones, the D. galeata clone, originates from an ephippium collected in the deep and large pre-alpine Lake Constance. In this lake, the seasonal period of $D$. galeata sexual reproduction coincides with the seasonal minimum of food quality (compare Wacker and Von Elert 2001 with Jankowski and Straile 2004). This suggests that this clone's parental clones were indeed adapted to use food quality as a trigger for their change in reproductive allocation. In contrast, our $D$. pulex clone was isolated from a pond. The similar induction of resting egg production upon low food quality in $D$. pulex suggests that food quality may be also an important trigger for obligate parthenogens in pond ecosystems.

It should be noted that although S. obliquus clearly is of lower food quality than Cryptomonas sp., S. obliquus also is a rather high-quality food for Daphnia. In fact, many studies use $S$. obliquus as a standard food when studying the physiology or life history of Daphnia. Not surprisingly, S. obliquus was also used in the majority of studies analysing resting egg production in Daphnia: a literature review revealed that $59.4 \%$ of all studies retrieved $(n=32)$ used S. obliquus as food. Furthermore, an additional $31.2 \%$ used other green algae, leaving only $6.25 \%$ using non-green algae or mixtures of different algal species, whereas only one recent study (3.1\%) used Cryptomonas sp. or other cryptomonads (see Electronic supplementary material, Appendix S1 for methods and references). This suggests that the vast majority of studies on Daphnia resting egg production were conducted with a hidden treatment, i.e. low food quality. Thus it is unclear if the quantity and quality of cues used to induce ephippia production in these studies would be sufficient to also induce ephippia production when Daphnia is fed food of a better quality, e.g. Cryptomonas sp. Consequently, the results of these studies with regard to the rate of ephippia production in response to other biotic and abiotic stressors should be re-evaluated. Only one study within our literature research also used, besides Scenedesmus obliquus, Cryptomonas sp. as a food source (Abrusán et al. 2007). The results of this study show that polyunsaturated fatty acids, especially eicosapentaenoic acid (EPA), are also important for the formation of resting eggs. Using strongly limiting food concentrations as an inductor for resting egg formation, Abrusán et al. (2007) showed that supplementation with EPA or even a Cryptomonas sp. diet of mothers, enhanced ephippia production in the offspring generation compared to a pure $S$. obliquus diet. This suggests that under severe food limitation EPA acts as a resource enabling the production of resting eggs, whereas with higher food availability, proteins act as a cue inducing a switch towards resting egg production. Hence, food quantity might cause a reversal in the food quality effect on ephippia production in Daphnia.

Nevertheless, our study and numerous other ones using S. obliquus as a food source (see Fig. S1) show that under less severe food limitation, the quality of $S$. obliquus is clearly sufficient to produce resting eggs. This suggests that Daphnia do not postpone resting egg production in situ until periods of severe food limitation, but instead use environmental cues such as density, photoperiod, and also food quality to initiate resting egg production in time.

In conclusion, our study suggests that food quality can trigger the reproductive mode in Daphnia. Supplementation experiments demonstrated that the absence of lipids and especially proteins triggered the switch to sexual reproduction and resting egg production. The observation that periods of minimal food quality coincide with the time of preferred ephippia production (sexual reproduction) in natural habitats suggests that a change in food quality is a major trigger for the onset of sexual reproduction/resting egg production in Daphnia in nature and enables Daphnia to fine-tune the optimal timing of sexual reproduction/ resting egg production. The timing of sexual and resting egg production must be under strong natural selection, as diapause is an essential component of the life cycle in Daphnia and many other animals and plants.

Acknowledgements We thank Walter Salzburger, Karl Otto Roth haupt and two anonymous referees for helpful comments on previous versions of this manuscript. Petra Merkel, Christine Gebauer and Timo Basen are thanked for help in the laboratory. We declare that all experiments comply with the current law of Germany.

\section{References}

Abrusán G, Fink P, Lampert W (2007) Biochemical limitation of resting egg production in Daphnia. Limnol Oceanogr 52:1724 1728

Ahlgren G, Lundstedt L, Brett MT, C Forsberg C (1990) Lipid composition and food quality of some freshwater phytoplankton for cladoceran zooplankters. J Plankton Res 12:809 818

Ahlgren G, Gustafsson I B, Boberg M (1992) Fatty acid content and chemical composition of freshwater microalgae. J Phycol 28:37 50

Alekseev V, Lampert W (2001) Maternal control of resting egg production in Daphnia. Nature 414:899 901

Cáceres C (1998) Interspecific variation in the abundance, production, and emergence of Daphnia diapausing eggs. Ecology 79:1699 1710 
Conover WJ, Iman RL (1981) Rank transformations as a bridge between parametric and non parametric statistics. Am Stat $35: 124128$

Deng HW (1996) Environmental and genetic control of sexual reproduction in Daphnia. Heredity 76:449 458

Dixon AFG, Kundu R (1998) Resource tracking in aphids: pro grammed reproductive strategies anticipate seasonal trends in habitat quality. Oecologia 114:73 78

Dixon AFG, Wellings PW, Carter C, Nichols JFA (1993) The role of food quality and competition in shaping the seasonal cycle in the reproductive activity of the sycamore aphid. Oecologia 95:89 92

Gilbert JJ (1981) Control of morphotype frequency distributions in populations of the rotifer Asplanchna sieboldi: factors influenc ing the production of the tocopherol dependent cruciform and campanulate morphotypes. Ecology 62:1299 1310

Gilbert JJ (2007) Timing of diapause in monogonont rotifers: dietary tocopherol. In: Alekseev VR, De Stasio B, Gilbert JJ (eds) Diapause in aquatic invertebrates, theory and human use. Springer, Berlin Heidelberg New York, pp 1819

Gilbert JJ, Thompson GA Jr (1968) Alpha tocopherol control of sexuality and polymorphism in the rotifer Asplanchna. Science 159:734 736

Guillard RR (1975) Cultures of phytoplankton for feeding of marine invertebrates. In: Smith WL, Chanley MH (eds) Culture of marine invertebrate animals. Plenum, New York, pp 2960

Hairston NG Jr, Kearns CM (2002) Temporal dispersal: ecological and evolutionary aspects of zooplankton egg banks and the role of sediment mixing. Integr Comp Biol 42:481 491

Hairston NG Jr, Lampert W, Cáceres CE, Holtmeier CL, Weider LJ, Gaedke U, Fischer JM, Fox JA, Post DM (1999) Rapid evolution revealed by dormant eggs. Nature 401:446

Hobæk A, Larsson P (1990) Sex determination in Daphnia magna. Ecology 71:2255 2268

Jankowski T, Straile D (2004) Allochronic differentiation among Daphnia species, hybrids and backcrosses: the importance of sexual reproduction for population dynamics and genetic archi tecture. J Evol Biol 17:312 321

Jüttner F, Leonhardt J, Möhren S (1983) Environmental factors affecting the formation of mesityloxide, dimethylallylic alcohol and other volatile compounds excreted by Anabaena cylindrica. J Gen Microbiol 129:407 412

Kleiven OT, Larsson P, Hobæk A (1992) Sexual reproduction in Daphnia magna requires three stimuli. Oikos 65:197 206

LaMontagne J, McCauley E (2001) Maternal effects in Daphnia: what mothers are telling their offspring and do they listen? Ecol Lett 4:64 71
Lampert W, Trubetskova I (1996) Juvenile growth rate as a measure of fitness in Daphnia. Funct Ecol 10:631 635

Louette G, De Meester L (2005) High dispersal capacity of cladoceran zooplankton in newly founded communities. Ecology 86(2):353 359

Maynard Smith J (1978) The evolution of sex. Cambridge University Press, London

Müller Navarra DC, Brett MT, Liston AM, Goldman CR (2000) A highly unsaturated fatty acid predicts carbon transfer between primary producers and consumers. Nature 403:74 77

Peck JR, Yearsley J, Barreau G (1999) The maintenance of sexual reproduction in a structured population. Proc R Soc Lond B Biol Sci 266:1857 1863

Schwartz SS, Hebert PD (1987) Methods for the activation of the resting eggs of Daphnia. Freshwater Biol 117:373 379

Simon J C, Rispe C, Sunnucks P (2002) Ecology and evolution of sex in aphids. Trends Ecol Evol 17:34 39

Slusarczyk M (1995) Predator induced diapause in Daphnia. Ecology 76:1008 1013

Sommer U (1985) Seasonal succession of phytoplankton in Lake Constance. Bioscience 35:351 357

Stearns SC (1992) The evolution of life histories. Oxford University Press, New York

Stross RG, Hill JC (1965) Diapause induction in Daphnia requires two stimuli. Science 150:1462 1464

Urabe J, Clasen J, Sterner RW (1997) Phosphorus limitation of Daphnia growth: is it real? Limnol Oceanogr 42:1436 1443

Von Elert E (2002) Determination of limiting polyunsaturated acids in Daphnia galeata using a new method to enrich food algae with single fatty acids. Limnol Oceanogr 47:1764 1773

Von Elert E, Wolffrom T (2001) Supplementation of cyanobacterial food with polyunsaturated fatty acids does not improve growth of Daphnia. Limnol Oceanogr 46:1552 1558

Von Elert E, Martin Creuzburg D, Le Coz JR (2003) Absence of sterols constrains carbon transfer between cyanobacteria and a freshwater herbivore (Daphnia galeata). Proc R Soc Lond B 270:1209 1214

Wacker A, von Elert E (2001) Polyunsaturated fatty acids: evidence for non substitutable biochemical resources in Daphnia galeata. Ecology 82:2507 2520

Weers PMM, Gulati RD (1997) Effect of the addition of polyunsat urated acids to the diet on the growth and the fecundity of Daphnia galeata. Freshwater Biol 38:721 729 


\author{
Appendix S1 \\ Electronic Supplementary Material for
}

Change in Food Quality Triggers Reproductive Mode in a Cyclical Parthenogen

\author{
Ulrike Koch*, Eric von Elert \& Dietmar Straile
}

${ }^{*}$ correspondence should be addressed to: Ulrike.Koch@uni-konstanz.de

This PDF file includes:

A literature survey of food algae used in experiments on the induction of Daphnia resting eggs

Fig. S1

References 
Appendix S1

Supporting Online Material

\section{A literature survey of food algae used in Daphnia resting egg induction experiments}

We searched the 'Web of Science' database for studies examining the induction of Daphnia resting egg production. Two different queries were used to identify the relevant literature:

1.) General search: "Daphnia AND resting egg OR ephippia OR ephippium" 211 results found

2.) Cited reference search: Stross RG and Hill JC (1965)

82 results found

Both queries revealed many studies which did not experimentally address resting egg production in Daphnia. Therefore, we screened all results and finally retained 25 (Numbers 1-25; chronological order) studies reporting experimental work of Daphnia resting egg induction. Our data base revealed an additional 6 studies (Number 26-31; chronological order) that were not found with the two queries. Hence, we report here on the food used in 32 studies of Daphnia resting egg induction.

In the majority (59.4\%) of studies Scenedesmus was used as food algae (Fig. S1). An additional $31 \%$ percent of studies used other green algae species, i.e.

Chlamydomonas, Chlorella or Selenastrum. In $6.25 \%$ of the studies Daphnia were 
fed with mixtures of various algal and bacteria species or non-algal food. Only in one study (3.1\%) Cryptomonas or another cryptophyte was used as food source.

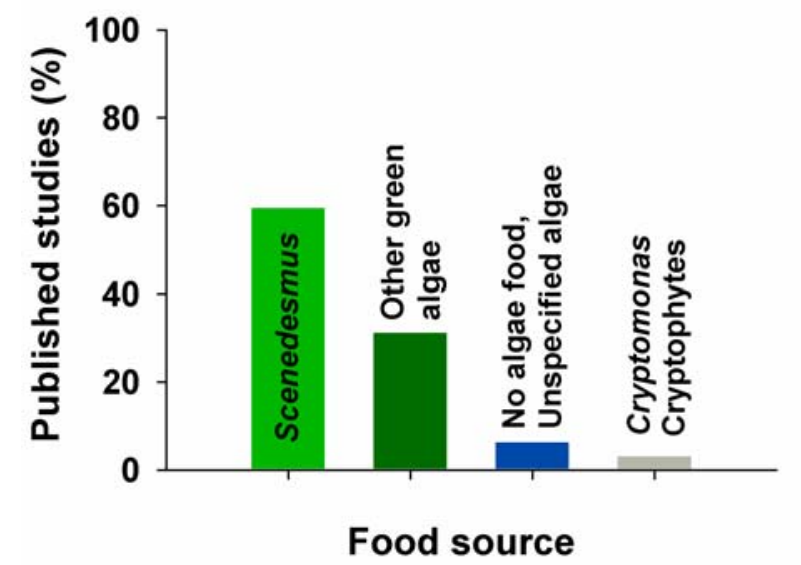

Fig. S1: Distribution of algae used in published studies on the induction of production of resting eggs by Daphnia.

\section{References}

1. Bernot RJ, Dodds WK, Quist MC, Guy CS (2006) Temperature and kairomone induced life history plasticity in coexisting Daphnia. Aquatic Ecology 40:361-372

2. Fitzsimmons JM, Innes DJ (2006) Inter-genotype variation in reproductive response to crowding among Daphnia pulex. Hydrobiologia 568:187-205

3. Duncan AB, Mitchell SE, Little TJ (2006) Parasite-mediated selection and the role of sex and diapause in Daphnia. J Evolution Biol 19:1183-1189

4. Lass S, Vos M, Wolinska J, Spaak P (2005) Hatching with the enemy: Daphnia diapausing eggs hatch in the presence of fish kairomones. Chemoecology 15:7-12

5. Slusarczyk M, Dawidowicz P, Rygielska E (2005) Hide, rest or die: a light-mediated diapause response in Daphnia magna to the treat of fish predation. Freshwater Biol 50: 141-146

6. Arbaciauskas K (2004) Life-history characteristics and fitness in descendents of parthenogenetic and ex-ephippio females of Daphnia magna. Hydrobiologia 526:211-218 Sp Iss

7. Slusarczyk M, Rygielska E (2004) Fish faeces as the primary source of chemical cues inducing fish avoidance diapause in Daphnia magna. Hydrobiologia 526:231-234 Sp Iss

8. Dzialowski AR, Lennon JT, O'Brien WJ, Smith VH (2003) Predator-induced phenotypic plasticity in the exotic cladoceran Daphnia lumholtzi. Freshwater Biol. 48:1593-1602

9. Lurling M, Roozen F, Van Donk E, Goser B (2003) Response of Daphnia to substances released from crowded congeners and conspecifics. J Plankton Res 25:967-978 
10. Alekseev V, Lampert W (2001) Maternal control of resting-egg production in Daphnia. Nature 414:899-901

11. Olmstead AW, LeBlanc GA (2001)Temporal and quantitative changes in sexual reproductive cycling of the cladoceran Daphnia magna by a juvenile hormone analog. J Exp Zool 290:148155

12. Slusarczyk M (2001) Food threshold for diapause in Daphnia under the threat of fish predation. Ecology 82:1089-1096

13. LaMontagne JM, McCauley E (2001) Maternal effects in Daphnia: What mothers are telling their offspring and do they listen? Ecol Lett 4:64-7

14. Boersma M, Boriss $\mathrm{H}$, Mitchell SE (2000). Maternal effects after sexual reproduction in Daphnia magna. J Plankton Res 22:279-285

15. Deng HW (1997) Photoperiodic response of sexual reproduction in the Daphnia pulex group is reversed in two distinct habitats. Limnol Oceanogr 42:609-611

16. Pijanowska J, Stolpe G (1996) Summer diapause in Daphnia as a reaction to the presence of fish. J Plankton Res 18:1407-1412

17. Deng HW (1996). Environmental and genetic control of sexual reproduction in Daphnia. Heredity $76: 449-458$

18. Spaak P (1995) Sexual reproduction in Daphnia - Interspecific differences in a hybrid species complex. Oecologia 104:501-507

19. Kleiven OT, Larsson P, Hobæk A (1992) Sexual reproduction in Daphnia magna requires three stimuli. Oikos 65:197-206

20. Yampolsky LY (1992) Genetic variation in the sexual reproduction rate within a population of a cyclical parthenogen, Daphnia magna. Evolution 46:833-837

21. Larsson $P$ (1991) Intraspecific variability in response to stimuli for male and ephippia formation in Daphnia pulex. Hydrobiologia 225:281-290

22. Hobæk A, Larsson P (1990) Sex Determination in Daphnia magna. Ecology 71:2255- 2268

23. Korpelainen $\mathrm{H}$ (1986) The effect of temperature and photoperiod on life history parameters of Daphnia magna (Crustacea: Cladocera). Freshwater Biol 16: 615-620

24. Carvalho GR, Hughes RN (1983) The effect of food availability, female culture density and photoperiod on ephippia production in Daphnia magna Straus (Crustacea, Cladocera) Freshwater Biol 13:37-46

25. Stross RG, Hill JC (1965). Diapause induction in Daphnia requires two stimuli. Science 150: 1462-1464

26. Abrusán G, Fink P, Lampert W (2007) Biochemical limitation of resting egg production in Daphnia. Limnol Oceanogr 52:1724-1728

27. Tessier AJ, Caceres CE (2004) Differentiation in sex investment by clones and populations of Daphnia. Ecol Lett 7:695-703

28. Spaak P, Boersma M (2001) The influence of fish kairomones on the induction and vertical distribution of sexual individuals of the Daphnia galeata species complex. Hydrobiologia 442: 185-193 
29. Berg LM, Pálsson S, Lascoux M (2001) Fitness and sexual response to population density in Daphnia pulex. Freshwater Biol 46:667-677

30. Innes DJ, Singleton DR (2000) Variation in allocation to sexual and asexual reproduction among clones of cyclically parthenogenetic Daphnia pulex (Crustacea: Cladocera). Biol J Linn Soc 71:771-787

31. Innes DJ, Dunbrack LD (1993) Sex allocation variation in Daphnia pulex. J Evolution Biol 6: 559-575

32. Stross RG (1966) Light and temperature requirements for diapause development and release in Daphnia. Ecology 47:368-374 